\title{
The Origin of Elements Heavier than Iron: in Honour of the 70th Birthday of Roberto Gallino
}

\author{
John Lattanzio and Maria Lugaro \\ Centre for Stellar and Planetary Astrophysics, School of Mathematical Sciences, \\ Building 28, Monash University, Victoria 3800, Australia
}

The origin of the elements is one of the major questions of the 21 st century. Our current knowledge dates back to the seminal papers of Hoyle (1954), Burbidge, Burbidge, Fowler and Hoyle (1957) and Cameron (1957). These papers defined the new field of nuclear astrophysics and put stellar nucleosynthesis on solid ground. They successfully identified, broadly, the sites and physics of the main processes producing the various elements.

It is well known that iron is the most stable element (as measured by the binding energy per nucleon). The process acting here is neutron capture, which has two idealised extremes: the rapid $(r)$ and slow $(s)$ neutron capture processes, where the rapid and slow refers to the relative speeds of neutron captures compared to $\beta$-decays.

With the development of modern fast computers, it has become possible to produce detailed models of stellar interiors, which provide the conditions for the expected nucleosynthesis. It has become clear that Asymptotic Giant Branch stars, the final stage in the evolution of most of stars, are producers of most of the $s$-process species. It is the study of these stars and their associated nucleosynthesis that is the principal topic of this special issue.

One of the main names associated with this field is Roberto Gallino, the author of over 350 papers on the subject. In the last 20 or so years, the contributions of Gallino and his co-workers have set the agenda and stimulated the research of the entire community.

Further, Roberto has been an inspiration to multiple generations of younger astrophysicists, to whom he has always been extremely generous with his time, ideas and enthusiasm. He (and Maurizio Busso) began the series of Torino Workshops, which have stimulated and fertilised the multi-disciplinary nature of this work.

For these reasons, a group of Roberto's collaborators and friends met to celebrate his work and review the current problems in the area of the origin of the elements heavier than iron. The meeting took place in Roberto's home city of Torino, in the hills where he

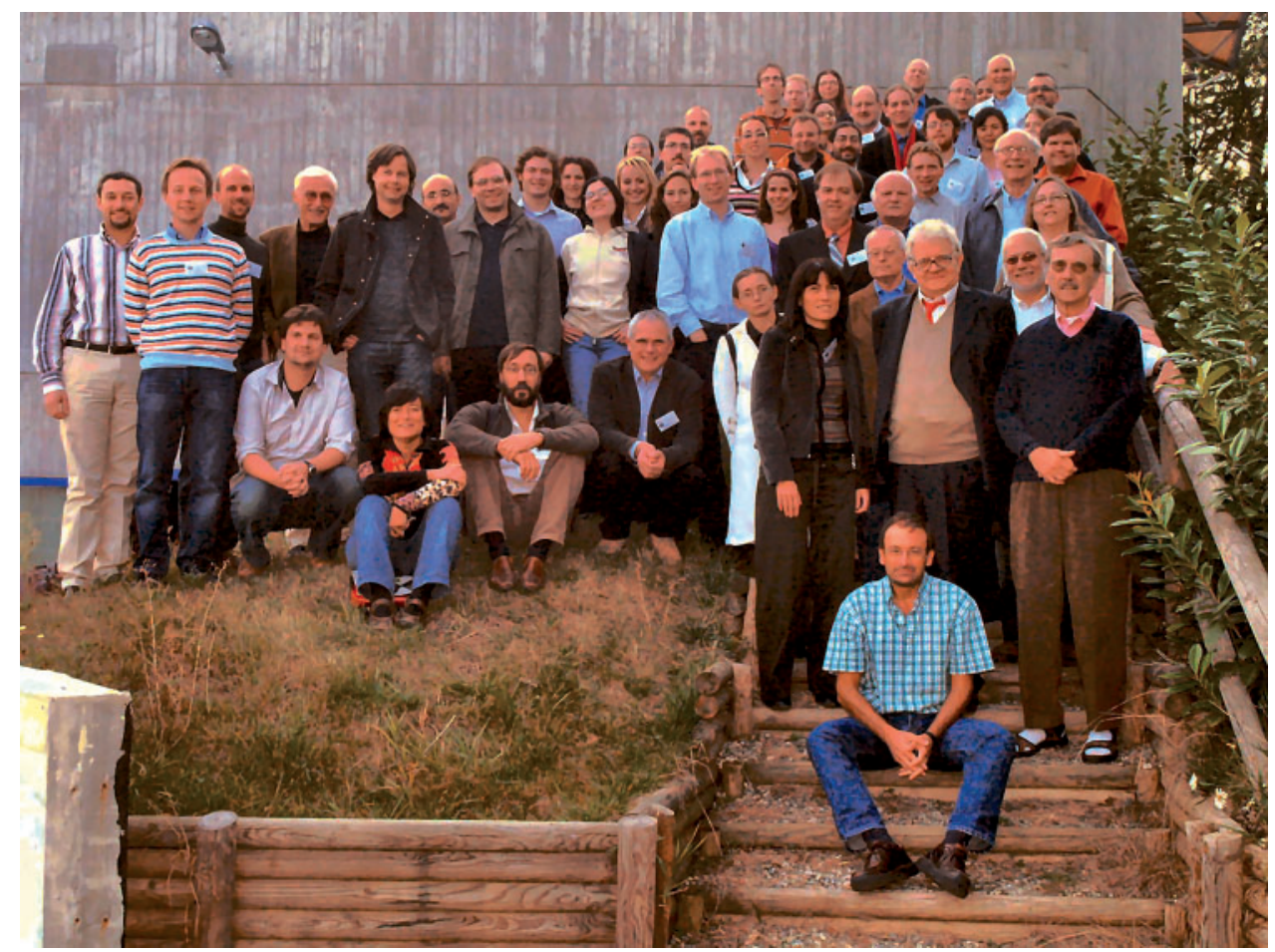


spent his childhood. Selected papers from that meeting are presented here as a record of the state of our understanding of how stars produce the heavy elements. This volume is multidisciplinary, covering topics from nuclear physics through planetary science and galactic chemical evolution. This multidisciplinarity is both a significant quality of any research on the origin of the elements as well as a thread in all Roberto's work.
We are proud to present these papers as a tribute to our colleague, friend and inspiration: Roberto Gallino.

\section{References}

Burbidge, E. M., Burbidge, G. R., Fowler, W. A. \& Hoyle, F., 1957, RvMP, 29, 547

Cameron, A. G. W., 1957, PASP, 69, 201

Hoyle, F., 1954, ApJS, 1, 121

\section{Supported by:}
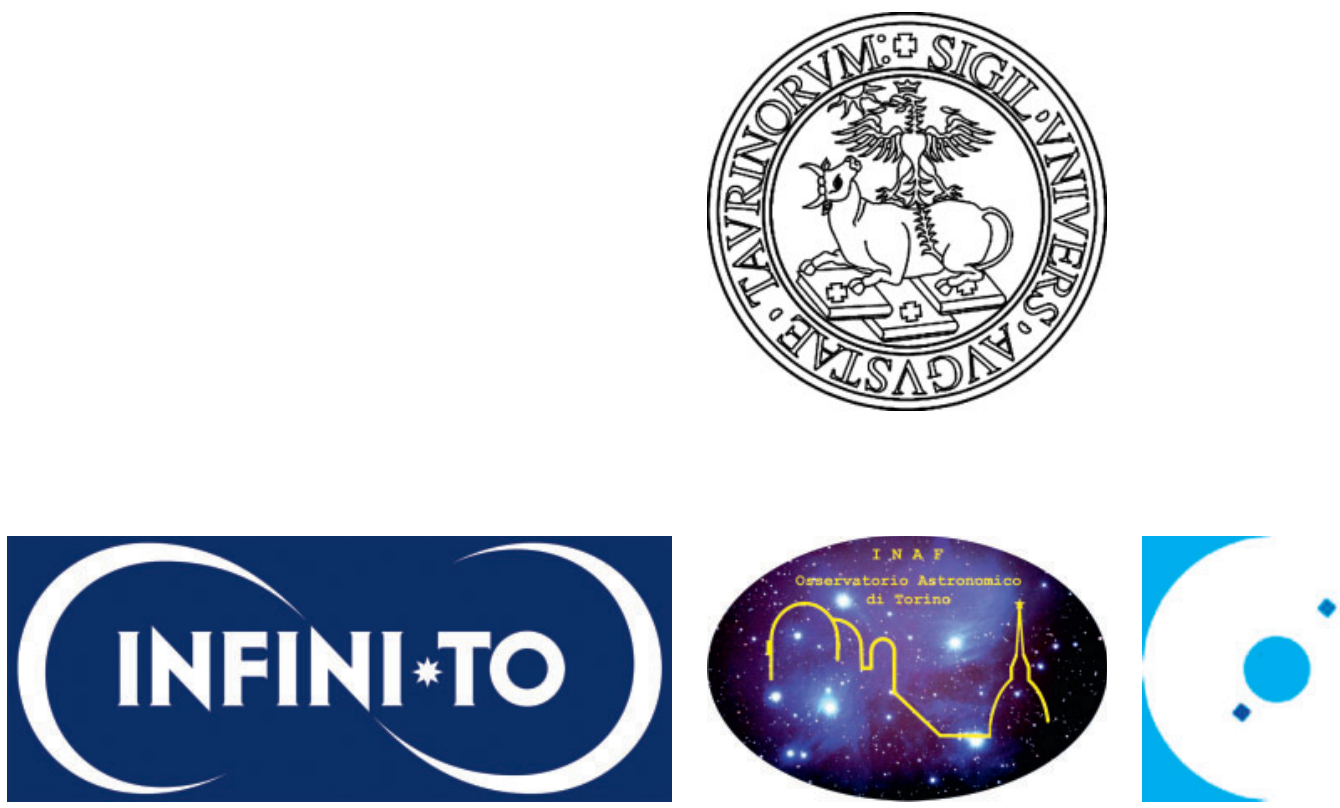

\section{IN A F}

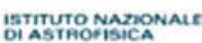
MATONA IBSTITUT

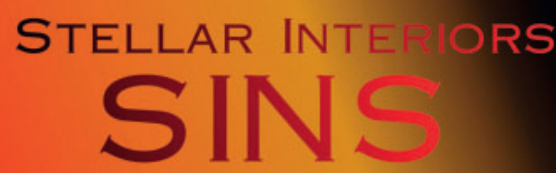

\& NUCLEOSYNTH SIS

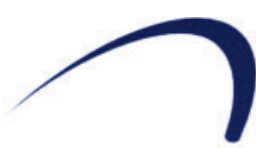

INFN 